\title{
Avoiding cross-sex friendships: The separability of people with and without cross-sex friends
}

\author{
Tobias Altmann ${ }^{1}$ (D) \\ Accepted: 13 September 2021 \\ (c) The Author(s) 2021
}

\begin{abstract}
Prior studies on individual differences in the preference for cross-sex friendships found that this preference was not normally distributed but was instead bimodal. In one group of people, the preferences for higher or lower proportions of cross-sex friendships appear to be normally distributed, whereas in a second and unexpectedly large group of people, the preference for cross-sex friends is exactly zero. If the people in the second group with no cross-sex friends at all actively avoid forming cross-sex friendships, then these individuals may be expected to differ systematically and meaningfully from individuals who report having at least one cross-sex friend. The present study tests this hypothesis. The Big Five, homophobia, physical attraction to the opposite sex, and demographic variables from a data set of 491 adult participants were used as potential predictors of group membership. Results showed that most predictors except the Big Five contributed to supporting the separability of the two groups. Findings are discussed with regard to the differentiation between close and general friends and the potential influence of cultural factors.
\end{abstract}

Keywords Cross-sex friendship $\cdot$ Heterosociality $\cdot$ Big five $\cdot$ Homophobia $\cdot$ Attraction

\section{Introduction}

Friendships are an important part of people's social lives and substantially influence an individual's quality of life and life satisfaction (Demir \& Weitekamp, 2007; Sheldon \& Hoon, 2006). Thus, who we choose to be friends with should be relevant to our quality of life as well. Research in social and personality psychology has provided insights into the mechanisms of how people choose their friends and which factors potentially affect friendship formation and maintenance (for an overview, see Harris \& Vazire, 2016). Consistent evidence has been found for the principle of homophily, that is, people's tendency to like and befriend others who are similar to themselves across a variety of attitudes as well as person and personality characteristics (Byrne, 1961; Fournet \& Barrat, 2014; McPherson et al., 2001). With respect to homophily based on biological sex, studies have shown that most people tend to prefer and feel closer to friends of the same sex (same-sex friends) as opposed to friends of the

Tobias Altmann

tobias.altmann@uni-due.de

1 Department of Psychology, University of Duisburg-Essen, Universitaetsstr. 2, 45141 Essen, Germany opposite sex (cross-sex friends; Baumgarte \& Nelson, 2009). Although this tendency appears to be consistent, there are also substantial interindividual differences in these friendship preferences (Altmann \& Roth, 2020; Reeder, 2003).

Only a few studies have explored these interindividual differences in the preferences for same-sex or cross-sex friendships (homo- or heterosociality). The predictors used in these studies can be categorized into three clusters: (a) person or demographic characteristics, (b) personality factors, and (c) attraction. First, preferences for cross-sex friendships are related to the person or demographic characteristics of biological sex, relationship status, and cultural background (Altmann \& Roth, 2020; Lönnqvist et al., 2014; Milardo, 1982; O'Meara, 1989). The aforementioned studies have shown that preferences for cross-sex friends can differ between men and women (Lönnqvist et al., 2014, however, cf. Altmann \& Roth, 2020) with generally higher scores for men. Changes in relationship status were shown to entail changes in the structure of the social network (Milardo, 1982), and being in a relationship potentially leads to lower rates of cross-sex friends (Altmann \& Roth, 2020) because cross-sex friends might be considered a relationship threat (Bleske-Rechek et al., 2012). Individual differences may also be attributable to cultural background with specific 
cultural rules of conduct about friendship and interactions with the opposite sex (Altmann \& Roth, 2020; Baumgarte, 2016; O'Meara, 1989). Of course, there is a rich literature in cultural psychology on the factors that influence general friendship formation processes (Adams \& Plaut, 2003; Baumgarte, 2016; Gareis, 1995). According to this literature (for an overview, see Altmann, 2021), cross-sex friendship formation processes may well be expected to also depend on cultural influences. However, studies on the topic of cultural influences on cross-sex friendship formation processes in particular are still lacking.

Second, personality factors may contribute to explaining the formation of cross-sex friendships. Previous studies have most frequently applied the Big Five personality factors (Altmann \& Roth, 2020; Harris \& Vazire, 2016; Laakasuo et al., 2016; Lönnqvist et al., 2014) on the basis of the argument that the Big Five represent a basic personality structure that has to be taken into account. These previous studies have shown that Openness and to some extent Neuroticism and Conscientiousness predict greater preferences for cross-sex friends. The findings of these few existing previous studies have been somewhat inconsistent with respect to the other Big Five factors, potentially due to the high level of diversity in how friends were sampled across the studies (e.g., Facebook contacts as opposed to close personal friends). All five factors may be relevant, but which factors dominate as predictors in each case may depend on the context of the study.

Apart from this general approach to personality, especially same-sex friendships have also been discussed in relation to homophobia (cf. O'Meara, 1989). On the one hand, homophobia might be associated with lower rates of same-sex friends on the basis of the reasoning that homophobic individuals will try to avoid behaviors that may be interpreted as homosexual and will hence try to avoid having close relationships with same-sex friends (Theodore $\&$ Basow, 2000). On the other hand, homophobia may be related to higher rates of same-sex friends on the basis of the reasoning that men try to avoid giving the impression that they are soft or feminine, and that women try to avoid giving the impression that they are hard or masculine. People may therefore avoid having close friendships with the opposite sex. Interestingly, for men, both hypotheses have been supported by empirical data (Galupo, 2009; Martino, 2000). Also interestingly, previous studies in this regard have focused almost exclusively on men so that research on women is essentially missing. In sum, further studies are needed to clarify the direction of the relation in women and men.

Third, physical attraction to the opposite sex is considered a strong influence of cross-sex friendships (Afifi \& Faulkner, 2000; Bleske \& Buss, 2000; Bleske-Rechek et al., 2012; Halatsis \& Christakis, 2009; Reeder, 2000). The aforementioned studies have supported the assumption that feelings of physical attraction in cross-sex friendships tend to be higher for men than for women. Findings by Bleske-Rechek and colleagues (Bleske \& Buss, 2000; Bleske-Rechek et al., 2012) have also shown that the costs of physical attraction are usually considered greater than the benefits by both women and men alike. Thus, a greater individual tendency to feel physically attracted to one's cross-sex friends may be associated with greater tension and conflict within one's cross-sex friendship dyads. Viewing a cross-sex friend as a potential sex partner may therefore be considered potentially inhibitive to selecting a cross-sex person as a friend. However, men typically report both a greater tendency to feel physically attracted to their cross-sex friends (BleskeRechek et al., 2012) and higher numbers and proportions of cross-sex friends compared with women (as described above; e.g., Lönnqvist et al., 2014). Therefore, physical attraction may also be an important reason for initiating a cross-sex friendship in the first place, at least for men (Bleske-Rechek \& Buss, 2001). Again, further research is needed.

In a comprehensive attempt to explain interindividual differences in same-sex versus cross-sex friendship preferences using a variety of predictors in a joint model, Altmann and Roth (2020) focused on heterosociality as defined by the individual proportion of cross-sex friends relative to the individual total number of friends (thus ranging from 0 to 1 ). Their primary findings indicate that both person and personality characteristics explain variance in the preferences. As a secondary but nonetheless important finding of their study, heterosociality did not appear to be normally distributed but was instead bimodal. For those participants who reported having (one or more) cross-sex friends, the distribution of heterosociality (cross-sex divided by total) was approximately normal for the scores, principally ranging from 0.01 to 1.00 . However, participants with a heterosociality score of exactly 0.00 were a substantially larger group than would have been expected if the whole scale had been normally distributed. Such a bimodal distribution may indicate that different mechanisms of friendship formation are at play in the two groups of participants. The question of whether this second group is indeed a distinct group was also posed by Altmann and Roth (2020). However, they excluded all participants with a heterosociality score of 0.00 from their analyses so that this question has remained an unanswered speculation.

\section{Goal and Hypotheses of the Present Study}

The goal of the present study was to test the speculation that individuals reporting zero cross-sex friends are likely to differ from people reporting more than zero cross-sex friends. To show this, two assumptions would have to be supported by the data. First, a strict test would compare individuals 
with zero cross-sex friends against individuals with only slightly more than zero cross-sex friends as the most conservative test. These two groups would be very similar with respect to their numbers of cross-sex friends (e.g., zero vs. one or two as opposed to, e.g., zero vs. 10). Therefore, any significant differences between these two groups would support the assumption that individuals reporting zero cross-sex friends systematically differ from individuals reporting at least one (more than zero) cross-sex friends. Second, such differences would then need to be absent between individuals reporting $X$ cross-sex friends versus individuals reporting $X+1$ cross-sex friends where $X$ is larger than one (e.g., comparing participants reporting three vs. participants reporting four cross-sex friends). Both assumptions would have to be fulfilled to support the hypothesis of the separability of the two groups. Accordingly, the present study tested this hypothesis with respect to person and personality factors as well as physical attraction on the basis of the previous research described above.

As detailed above, there has not been much research on cross-sex friendship in general (e.g., with respect to crosscultural influences) or more specifically on the avoidance of cross-sex friendship. Therefore, for the present study, it was necessary to adopt a partially exploratory approach. To build the present hypotheses, findings from previous studies on interindividual differences as predictors of preferences for cross-sex friendships in general (as explicated above) were applied to the present research question. In light of the research reviewed above, we generally expected to find that all potential predictors used in the previous studies (i.e., sex, relationship status, migration background, the Big Five, homophobia, and being physically attracted to one's crosssex friends) would also be potentially relevant and therefore significant predictors of having zero versus having one or more cross-sex friends. Additionally, with respect to the second assumption described above, we expected to find that all predictors would be nonsignificant when comparing participants with $X$ versus $X+1$ cross-sex friends when $X$ was larger than one.

\section{Method}

\section{Sample and Procedure}

The data analyzed in this study were collected by having the department staff and student assistants distribute leaflets on a local university campus located in Germany. Participants completed the questionnaires individually or in group sessions (paper and pencil assessment) in exchange for 5 Euro (approximately \$6 US) as compensation. All procedures were in accordance with the local ethical guidelines.
Three participants were excluded from the analyses because they indicated that their mastery of the German language was less than very good and fluent (rating options were excellent/mother tongue, very good/fluent, good [small talk level], mediocre, and sufficient). Participants with more than 15 close friends $(n=5)$ or more than 30 general friends $(n=6)$ were also excluded for reasons of plausibility. These participants probably misread or misunderstood the definitions of friendship provided in the booklet.

The final sample consisted of 378 women and 113 men. Their mean age was 25.1 years $(S D=7.3)$, ranging from 18 to 67 . The sample was predominantly a student sample with $86.6 \%$ reporting "student" as their primary occupation status, whereas $12.2 \%$ reported being employed, and $1.2 \%$ reported being unemployed. A total of $58.0 \%$ reported that they were in a stable relationship. Migration background was defined as having at least one parent who was born abroad, which was confirmed by $38.9 \%$ of participants. Of those with a migration background, the largest group reported Turkey as their country of origin (35\%). The diversity of countries of origin was surprisingly large (40 different countries) so that the variable could not meaningfully be used as a moderator if each country had been coded individually. Therefore, only the first dichotomous variable was used to differentiate between people with and people without a migration background. Thus, the variable represents potential specificities of the German culture.

\section{Measures}

\section{Numbers of Cross-Sex Friends}

Participants first read definitions of close and general friends that were to be used when answering the survey (Altmann, 2020; Stehlé et al., 2013). Close friends were defined as people they see often and share intimate information with. General friends were defined as people with whom they meet less often but still regularly share private but not intimate details with. Participants were asked to exclude relatives, friends who are solely online friends, and sex partners. Participants were then instructed to think about their friends as defined above and to write down the names of their close friends and their general friends on separate lists. They were then instructed to count and report the numbers of men and women on both lists separately.

\section{Big Five}

The German version of the NEO Five Factor Inventory (NEO-FFI; Borkenau \& Ostendorf, 2008) was used to assess the Big Five. The NEO-FFI contains 60 items for assessing Agreeableness, Conscientiousness, Extraversion, 
Neuroticism, and Openness, rated on a 5-point Likert scale. Cronbach's alpha scores ranged from 0.75 for Agreeableness to 0.86 for Neuroticism.

\section{Homophobia}

The Homophobia Scale (HPS; Wright et al., 1999) was used to assess homophobia. The HPS is a 25 -item inventory for assessing negative attitudes, feelings, thoughts, and actions toward or against homosexual people using a 5-point Likert scale. Cronbach's alpha for the total score was 0.78 . The original items were translated in a prior research project.

\section{Physical Attraction}

To assess participants' tendency to feel physically attracted to their cross-sex friends, a new item was created. The English translation of the item reads "I have or have had erotic thoughts about some of my friends of the opposite sex." The item was rated on a 10-point Likert scale ranging from $1=$ with (almost) none of them to $10=$ with (almost) all of them.

\section{Results}

On average, participants reported $8.8(S D=6.9)$ cross-sex friends and $15.8(S D=9.0)$ same-sex friends. These numbers are in line with previous studies showing a general preference for same-sex friends (Baumgarte \& Nelson, 2009; Reeder, 2003). Out of participants' cross-sex friends, the numbers of close and general friends were $2.3(S D=2.2)$ and $6.5(S D=5.7)$, respectively, on average.

Using these data, two sets of two dichotomous variables were created. The first set was used to test the first assumption (differences between participants with zero versus one or more cross-sex friends), whereas the second set was used to test the second assumption (absence of such differences between participants with $\mathrm{X}$ vs. $\mathrm{X}+1$ cross-sex friends). For the first variable from the first set (labeled CSF-Close), participants were assigned the value " 0 " if they reported having zero cross-sex friends among their close friends. Participants who reported having more than zero but less than the average (i.e., 2.3, see above) were assigned the value "1" on this variable. All other cases were treated as missing $\left(n_{\text {CSF-Close }}=309\right)$. The second variable from the first set (labeled CSF-General) was created analogously with respect to participants' general friends (using the respective average of 6.5, see above; $n_{\text {CSF-General }}=316$ ). With respect to CSFClose, 105 (33.4\%) participants reported zero close crosssex friends. However, with respect to CSF-General, only 27 (8.5\%) participants reported zero general cross-sex friends, potentially limiting reliability (this issue is elaborated on in the Discussion section).

To test whether participants with $\mathrm{X}$ versus $\mathrm{X}+1$ (when $\mathrm{X}$ was larger than zero) cross-sex friends differed comparably as a cross-check, the second set of dichotomous variables labeled CSF-Close-X and CSF-General-X was created correspondingly. $\mathrm{X}$ was set equal to the mean numbers of close and general cross-sex friends (2.3 and 6.5, respectively): Participants with two cross-sex friends were assigned the value " 0 ," and participants with three crosssex friends were assigned the value " 1 " in the variable CSF-Close- $X$, that is, the whole numbers surrounding the mean of $2.3\left(n_{\text {CSF-Close- } X}=174\right)$. This was repeated for CSFClose- $\mathrm{X}$ with six and seven cross-sex friends, respectively, that is, the whole numbers surrounding the mean of 6.5 $\left(n_{\text {CSF-General- } \mathrm{X}}=46\right)$.

\section{Regression Analyses}

To test the hypothesis that the two groups that had or did not have cross-sex friends are differentiable, a hierarchical binary logistic regression with the two variables CSF-Close and CSF-General as dependent variables and the person characteristics, personality characteristics, and attraction as predictors was used. Predictors were entered in three steps, according to the three clusters of potential predictors discussed in the Introduction. In the first step, person and sociodemographic variables (sex, relationship status, and migration background) were entered. In the second step, the personality variables (Big Five: NEO-FFI, homophobia: HPS) were entered. In the third step, the physical attraction variable was entered. Results are presented in Table 1 for CSF-Close and in Table 2 for CSF-General.

With respect to CSF-Close, homophobia and physical attraction remained significant predictors in Step 3 when controlling for all other variables in the model. Controlling for all other variables, a difference of 1 point on the 5-point Likert scale used to measure homophobia (HPS) was associated with a $47 \%$ decrease in the likelihood of having cross-sex friends among one's close friends. Analogously, controlling for all other variables, a difference of 1 point on the 10-point scale used to measure the physical attraction variable was associated with a decrease of $9 \%$ in the likelihood of having cross-sex friends among one's close friends.

With respect to CSF-General, relationship status and migration background were the only significant predictors in all steps. Participants who were in a relationship or reported having a migration background were $73 \%$ and $67 \%$ less likely to have cross-sex friends among their general friends, respectively.

Mother tongue is a variable that can be used as an alternative to migration background to potentially represent the influence of the heritage culture. It has also been assessed 
Table 1 Hierarchical logistic regression results for having versus not having close cross-sex friends

\begin{tabular}{|c|c|c|c|c|}
\hline Variable & B & SE & Wald's $\chi^{2}$ & Odds ratio \\
\hline \multicolumn{5}{|l|}{ Step 1} \\
\hline Constant & $1.64 *$ & 0.81 & 4.06 & \\
\hline Migration & -0.36 & 0.25 & 2.08 & 0.70 \\
\hline Relationship status & -0.17 & 0.25 & 0.47 & 0.84 \\
\hline Sex & -0.11 & 0.30 & 0.13 & 0.90 \\
\hline \multicolumn{5}{|l|}{ Step 2} \\
\hline Constant & $3.29 *$ & 1.52 & 4.68 & \\
\hline Migration & -0.06 & 0.28 & 0.05 & 0.94 \\
\hline Relationship status & -0.24 & 0.25 & 0.88 & 0.79 \\
\hline Sex & -0.18 & 0.34 & 0.27 & 0.84 \\
\hline Agreeableness & -0.10 & 0.15 & 0.46 & 0.90 \\
\hline Conscientiousness & -0.18 & 0.18 & 1.02 & 0.84 \\
\hline Extraversion & 0.03 & 0.16 & 0.03 & 1.03 \\
\hline Neuroticism & -0.07 & 0.16 & 0.17 & 0.94 \\
\hline Openness & 0.07 & 0.16 & 0.21 & 1.07 \\
\hline Homophobia & $-0.63 * *$ & 0.24 & 6.83 & 0.54 \\
\hline \multicolumn{5}{|l|}{ Step 3} \\
\hline Constant & $4.41 * *$ & 1.64 & 7.23 & \\
\hline Migration & -0.14 & 0.28 & 0.25 & 0.87 \\
\hline Relationship status & -0.34 & 0.26 & 1.65 & 0.72 \\
\hline Sex & -0.37 & 0.36 & 1.07 & 0.69 \\
\hline Agreeableness & -0.09 & 0.15 & 0.33 & 0.92 \\
\hline Conscientiousness & -0.21 & 0.18 & 1.38 & 0.81 \\
\hline Extraversion & 0.04 & 0.16 & 0.05 & 1.04 \\
\hline Neuroticism & -0.05 & 0.16 & 0.11 & 0.95 \\
\hline Openness & 0.05 & 0.16 & 0.09 & 1.05 \\
\hline Homophobia & $-0.63 * *$ & 0.24 & 6.87 & 0.53 \\
\hline Physical attraction & $-0.10^{*}$ & 0.05 & 4.25 & 0.91 \\
\hline
\end{tabular}

${ }^{*} p<.05 . * * p<.01$

whether participants spoke German or a different language as their first language and used this variable exploratorily instead of migration background. The results were largely the same as the results presented here for both dependent variables.

To test the $X$ versus $X+1$ cross-check, the analyses were repeated using CSF-Close-X and CSF-General-X (as described above) as dependent variables. Results were consistently nonsignificant with Wald's $\chi^{2}<0.66, p>0.417$ for CSF-Close-X and Wald's $\chi^{2}<3.13, p>0.077$ for CSF-General-X.

\section{Discussion}

The present study was designed to test the hypothesis that people who categorically avoid forming cross-sex friendships (i.e., individuals who report having no cross-sex
Table 2 Hierarchical logistic regression results for having versus not having general cross-sex friends

\begin{tabular}{lllll}
\hline Variable & $\mathrm{B}$ & SE & Wald's $\chi^{2}$ & Odds ratio \\
\hline Step 1 & & & & \\
Constant & $6.70^{* *}$ & 1.53 & 19.08 & \\
Migration & $-1.31^{* *}$ & 0.44 & 8.92 & 0.27 \\
Relationship status & $-1.36^{* *}$ & 0.49 & 7.61 & 0.26 \\
Sex & -0.03 & 0.53 & 0.00 & 0.98 \\
Step 2 & & & & \\
Constant & $5.42^{*}$ & 2.46 & 4.87 & \\
Migration & $-1.17 *$ & 0.49 & 5.73 & 0.31 \\
Relationship status & $-1.42^{* *}$ & 0.51 & 7.87 & 0.24 \\
Sex & -0.19 & 0.62 & 0.10 & 0.83 \\
Agreeableness & 0.28 & 0.25 & 1.29 & 1.33 \\
Conscientiousness & -0.10 & 0.32 & 0.10 & 0.90 \\
Extraversion & 0.34 & 0.27 & 1.61 & 1.41 \\
Neuroticism & 0.05 & 0.27 & 0.03 & 1.05 \\
Openness & 0.04 & 0.27 & 0.02 & 1.04 \\
Homophobia & -0.29 & 0.37 & 0.64 & 0.75 \\
Step 3 & & & & \\
Constant & 4.48 & 2.69 & 2.77 & \\
Migration & $-1.12^{*}$ & 0.50 & 5.08 & 0.33 \\
Relationship status & $-1.34 * *$ & 0.52 & 6.72 & 0.26 \\
Sex & 0.00 & 0.66 & 0.00 & 1.00 \\
Agreeableness & 0.27 & 0.25 & 1.21 & 1.32 \\
Conscientiousness & -0.06 & 0.33 & 0.04 & 0.94 \\
Extraversion & 0.33 & 0.27 & 1.49 & 1.39 \\
Neuroticism & 0.03 & 0.27 & 0.01 & 1.03 \\
Openness & 0.06 & 0.27 & 0.05 & 1.06 \\
Homophobia & -0.28 & 0.37 & 0.56 & 0.76 \\
Physical attraction & 0.08 & 0.10 & 0.64 & 1.08 \\
\hline
\end{tabular}

${ }^{*} p<.05 . * * p<.01$

friends) differ systematically from individuals who report having one or more cross-sex friends. This hypothesis was tested and cross-checked using person characteristics, personality characteristics, and physical attraction as potential differentiators.

\section{Predictors of the Avoidance of Close Cross-Sex Friends}

Homophobia was found to be the main determinant for avoiding close cross-sex friendships with a large effect size. A greater tendency to have negative thoughts and feelings about people with a homosexual orientation was associated with an increased likelihood of avoiding cross-sex friendships. This supports findings by Martino (2000), who stated that homophobia in men is related to a fear of appearing too feminine such that men tend to avoid close cross-sex friendships. Interestingly, Martino (2000) also found that 
homophobic men try to secure their views and beliefs by encouraging other men to comply with and follow their behavior. It may be interesting for future research to also explore the effects of the beliefs and attitudes of an individual's closest friends on the friendship choices of this individual (Parker et al., 2008). Research is also needed to clarify the role of homophobia in women in general as this is still a severely understudied phenomenon (Basow \& Johnson, 2000).

The second determinant in this domain was the tendency to feel physically attracted to one's close cross-sex friends. This finding was expected and is in line with previous research (especially Bleske \& Buss, 2000; Bleske-Rechek et al., 2012) that stated that physical attraction is subjectively associated with greater costs than benefits. Consistently, individuals who avoid cross-sex friendships reported more of a tendency to feel physically attracted to their cross-sex friends in the present study. Although a smaller effect, this attraction may be speculated to be a potential cause for some individuals to either approach a cross-sex peer as a potential mate or avoid the potential relationship altogether. This causal inference is of course speculative and not directly supported by the cross-sectional data from the present study.

In sum, with respect to close friends, individuals who avoid cross-sex friendships do not appear to differ with respect to the basic personality dimensions as conceptualized in the Five Factor Model or with respect to basic demographic characteristics. However, they may be described as more homophobic and as having more of a tendency to feel physically attracted to their cross-sex friends, factors that may partly explain their friendship choices.

\section{Predictors of the Avoidance of General Cross-Sex Friends}

Determinants of the avoidance of general cross-sex friends were relationship status and migration background with rather large effect sizes. As expected, individuals in a relationship were more likely to avoid forming cross-sex friendships. Such friends might be considered a relationship threat, and thus, it appears plausible that long-term changes in the friendship network favor the reduction of cross-sex friends (Bleske-Rechek et al., 2012; Milardo, 1982).

Individuals with a migration background were also more likely to avoid forming general cross-sex friendships. This finding may be explained by findings on the values of immigrants. Wakil et al. (1981) showed that immigrants in general were inclined to focus on and fortify their core values rather than adopt the values of the host country. Focusing on differences between cultures of origin, Arends-Toth and van de Vijver (2009) demonstrated that Turkish and Arabic cultures scored highest on traditional values. Considering these findings in connection with the fact that immigration in Germany is largely from Turkish and Arabic states, as was also found in the present sample, having a migration background was associated with a greater likelihood of avoiding cross-sex friends because this is an untraditional type of friendship (Bleske-Rechek et al., 2012). However, this finding was also (or perhaps even more so) expected for close cross-sex friends and not only for general cross-sex friends.

The same finding can also be interpreted from the perspective that the people without a migration background (i.e., Germans) may be more open to forming cross-sex friendships than people from other cultures. This finding might also be explained by the specifics of how friendship is defined and lived in Germany. The narrow cultural context of the present study is a noteworthy limitation (see below), and future studies are needed to understand cultural influences on cross-sex friendship formation (see Altmann, 2021).

In sum, with respect to general friends, individuals avoiding cross-sex friendships do not appear to differ with respect to the basic personality dimensions as conceptualized in the Five Factor Model or with respect to homophobia and physical attraction. However, they may also be in a relationship or may have a migration background (with respect to Germany), two factors that may also have an influence on their friendship choices.

A secondary finding of the present study is that the significant predictors differ between close and general friendships. These differences are likely due to the different procedures that must be followed to become a close or a general friend. A close friend will be selected on the basis of a person's individual criteria, such as the tendency to avoid a particular type of person, for instance, people of the opposite sex. By contrast, a person's general friends may likely also include the friends of one's close friends, colleagues at work, and so forth. Here, the mechanisms of individual selection may be less effective because, for instance, (cross-sex) colleagues are harder to avoid without causing conflicts at work. Therefore, it may be the case that close friends are typically being selected from the people in one's environment, whereas general friends are typically being accepted as the people in one's environment. Again, this finding may also be specific to the present sample from Germany where the distinction between close and general friends is common. In other countries or cultures, this distinction may be less relevant or less common, and thus, the predictors may depend on the type of friendship only in certain cultural contexts.

\section{Limitations}

There are several limitations that have to be considered when interpreting the present findings. Three of them will be elaborated on in the following: the bimodal distribution, the sample, and the cultural context. 
First, the hypothesis was based on the finding that the distribution of numbers of cross-sex friends in relation to total numbers of friends was not normal but was instead bimodal. This bimodality was indeed distinct with regard to participants' close friends, but it was less distinct with regard to general friends. This may indicate that the tendency to avoid forming cross-sex friendships is considerably stronger with respect to one's close friends than among one's other or more general friends. Therefore, the latter findings may be less reliable, and the focus on participants' close friends may be the more relevant focus. Replications are needed to confirm the validity of the present findings as well as the assumptions of bimodal distributions for both close and general friendships.

Second, the sample contained predominantly students so that findings cannot be generalized to other parts of society. Age as well as achieving a higher level of education may be associated with certain traits, such as conscientiousness and the need for cognition, which in turn may influence priorities with respect to friendship choices. The present findings are therefore limited to young and educated populations.

A third limitation lies in the narrow cultural context in which the study was conducted. As has been argued above and has often been shown in cross-cultural studies, the definitions, mechanisms, and individual experiences of friendship differ substantially between cultures (Adams \& Plaut, 2003; Baumgarte, 2016; Gareis, 1995). The study was conducted in Germany so that the findings may be limited to this country or this cultural region. This limitation pertains to several aspects of the present study, such as the basic definition of friendship, what kinds of relationships are considered "friendships," the relevance of the distinction between close and general friends, and the relevance of each predictor included in the present study. A variable to control for cultural influences was included in the model-at least it was expected to do so to some extent by capturing the potential specificity of the German culture. However, future studies could benefit from applying a more comprehensive approach to studying cross-cultural differences in cross-sex friendship formation processes.

\section{Conclusion}

The hypothesis that individuals with zero cross-sex friends differ from individuals with one, two, or more cross-sex friends was partly supported by the present findings. Contrary to the hypotheses, personality factors played no discernible role. However, individual differences in homophobia, physical attraction, relationship status, and migration background contributed to explaining differences between individuals who avoid and those who allow themselves to have cross-sex friends. The separability of the two groups suggests that future research may have to take this distinction into account. However, replications and research on crosscultural differences (see Baumgarte, 2016) are still needed.

Authors' Contributions Not applicable.

Funding Open Access funding enabled and organized by Projekt DEAL.

Data Availability Data are available via email from the author upon reasonable request.

Code Availability Not applicable.

\section{Declarations}

Conflicts of Interest/Competing Interests There is no conflict of interest to be reported.

Ethics Approval All procedures performed in this study involving human participants were in accordance with the ethical standards of the local institutional research committee and with the 1964 Helsinki declaration and its later amendments or comparable ethical standards.

Consent to Participate All participants in the study gave informed consent.

Consent for Publication All participants in the study gave informed consent.

Open Access This article is licensed under a Creative Commons Attribution 4.0 International License, which permits use, sharing, adaptation, distribution and reproduction in any medium or format, as long as you give appropriate credit to the original author(s) and the source, provide a link to the Creative Commons licence, and indicate if changes were made. The images or other third party material in this article are included in the article's Creative Commons licence, unless indicated otherwise in a credit line to the material. If material is not included in the article's Creative Commons licence and your intended use is not permitted by statutory regulation or exceeds the permitted use, you will need to obtain permission directly from the copyright holder. To view a copy of this licence, visit http://creativecommons.org/licenses/by/4.0/.

\section{References}

Adams, G., \& Plaut, V. C. (2003). The cultural grounding of personal relationship: Friendship in North American and West African worlds. Personal Relationships, 10(3), 333-347. https://doi.org/ 10.1111/1475-6811.00053

Afifi, W. A., \& Faulkner, S. L. (2000). On being "just friends": The frequency and impact of sexual activity in cross-sex friendships. Journal of Social and Personal Relationships, 17(2), 205-222. https://doi.org/10.1177/0265407500172003

Altmann, T. (2020). Distinctions in friendship research: variations in the relations between friendship and the Big Five. Personality and Individual Differences, 154, 109727. https://doi.org/10.1016/j. paid.2019.109727 
Altmann, T. (2021). Friendship in personality and cultural psychology: International perspectives. Nova Science.

Altmann, T., \& Roth, M. (2020). Individual differences in the preference for cross-sex friendship (heterosociality) in relation to personality. Personality and Individual Differences, 157. https://doi. org/10.1016/j.paid.2020.109838

Arends-Toth, J., \& van de Vijver, F. J. (2009). wakil. International Journal of Psychology, 44(3), 161-169. https://doi.org/10.1080/ 00207590701545676

Basow, S. A., \& Johnson, K. (2000). Predictors of homophobia in female college students. Sex Roles, 42, 391-404. https://doi.org/ 10.1023/A:1007098221316

Baumgarte, R. (2016). Conceptualizing cultural variations in close friendships. Online Readings in Psychology and Culture, 5(4). https://doi.org/10.9707/2307-0919.1137

Baumgarte, R., \& Nelson, D. W. (2009). Preference for same- versus cross-sex friendships. Journal of Applied Social Psychology, 39(4), 901-917. https://doi.org/10.1111/j.1559-1816.2009. 00465.x

Bleske, A. L., \& Buss, D. M. (2000). Can men and women be just friends? Personal Relationships, 7(2), 131-151. https://doi.org/ 10.1111/j.1475-6811.2000.tb00008.x

Bleske-Rechek, A. L., \& Buss, D. M. (2001). Opposite-sex friendship: Sex differences and similarities in initiation, selection, and dissolution. Personality and Social Psychology Bulletin, 27, 1310 1323. https://doi.org/10.1177/01461672012710007

Bleske-Rechek, A. L., Somers, E., Micke, C., Erickson, L., Matteson, L., Stocco, C., . . . Ritchie, L. (2012). Benefit or burden? Attraction in cross-sex friendship. Journal of Social and Personal Relationships, 29(5), 569-596. https://doi.org/10.1177/0265407512 443611

Borkenau, P., \& Ostendorf, F. (2008). NEO-Fünf-Faktoren Inventar (NEO-FFI) nach Costa und McCrae [NEO-Five-Factor Inventory (NEO-FFI) by Costa and McCrae]. Hogrefe.

Byrne, D. (1961). Interpersonal attraction and attitude similarity. Journal of Abnormal and Social Psychology, 62, 713-715.

Demir, M., \& Weitekamp, L. A. (2007). I am so happy cause today I found my friend: Friendship and personality as predictors of happiness. Journal of Happiness Studies, 8(2), 181-211. https://doi. org/10.1007/s10902-006-9012-7

Fournet, J., \& Barrat, A. (2014). Contact patterns among high school students. PLoS ONE, 9(9), e107878. https://doi.org/10.1371/journ al.pone. 0107878

Galupo, M. P. (2009). Cross-category friendship patterns: Comparison of heterosexual and sexual minority adults. Journal of Social and Personal Relationships, 26(6-7), 811-831. https://doi.org/10. $1177 / 0265407509345651$

Gareis, E. (1995). Intercultural friendship: A qualitative study. University Press of America Inc.

Halatsis, P., \& Christakis, N. (2009). The challenge of sexual attraction within heterosexuals' cross-sex friendship. Journal of Social and Personal Relationships, 26(6-7), 919-937. https://doi.org/10. $1177 / 0265407509345650$

Harris, K., \& Vazire, S. (2016). On friendship development and the Big Five personality traits. Social and Personality Psychology Compass, 10, 647-667. https://doi.org/10.1111/spc3.12287

Laakasuo, M., Rotkirch, A., Berg, V., \& Jokela, M. (2016). The company you keep: Personality and friendship characteristics. Social Psychological and Personality Science, 8(1), 66-73. https://doi. org/10.1177/1948550616662126
Lönnqvist, J.-E., Itkonen, J. V. A., Verkasalo, M., \& Poutvaara, P. (2014). The five-factor model of personality and degree and transitivity of Facebook social networks. Journal of Research in Personality, 50, 98-101. https://doi.org/10.1016/j.jrp.2014.03.009

Martino, W. (2000). Policing masculinities: Investigating the role of homophobia and heteronormativity in the lives of adolescent school boys. The Journal of Men's Studies, 8(2), 213-236. https:// doi.org/10.3149/jms.0802.213

McPherson, M., Smith-Lovin, L., \& Cook, J. M. (2001). Birds of a feather: Homophily in social networks. Annual Review of Sociology, 27(1), 415-444. https://doi.org/10.1146/annurev.soc.27.1.415

Milardo, R. M. (1982). Friendship networks in developing relationships: Converging and diverging social environments. Social Psychology Quarterly, 45(3). https://doi.org/10.2307/3033649

O'Meara, J. (1989). Cross-sex friendship: Four basic challenges of an ignored relationship. Sex Roles, 21(7-8), 525-543. https://doi.org/ 10.1007/BF00289102

Parker, S. L., Parker, G. R., \& McCann, J. A. (2008). Opinion taking within friendship networks. American Journal of Political Science, 52(2), 412-420. https://doi.org/10.1111/j.1540-5907.2008. 00320. $\mathrm{x}$

Reeder, H. M. (2000). "I like you ... as a friend": The role of attraction in cross-sex friendship. Journal of Social and Personal Relationships, 17(3), 329-348. https://doi.org/10.1177/0265407500 173002

Reeder, H. M. (2003). The effect of gender role orientation on sameand cross-sex friendship formation. Sex Roles, 49(3-4), 143-152. https://doi.org/10.1023/a:1024408913880

Schwartz, S. H. (1992). Universals in the content and structure of values: Theory and empirical tests in 20 countries. In M. Zanna (Ed.), Advances in Experimental Social Psychology (Vol. 25, pp. 1-65). Academic Press.

Sheldon, K. M., \& Hoon, T. H. (2006). The multiple determination of well-being: Independent effects of positive traits, needs, goals, selves, social supports, and cultural contexts. Journal of Happiness Studies, 8(4), 565-592. https://doi.org/10.1007/ s10902-006-9031-4

Stehlé, J., Charbonnier, F., Picard, T., Cattuto, C., \& Barrat, A. (2013). Gender homophily from spatial behavior in a primary school: A sociometric study. Social Networks, 35(4), 604-613. https://doi. org/10.1016/j.socnet.2013.08.003

Theodore, P. S., \& Basow, S. A. (2000). Heterosexual masculinity and homophobia: A reaction to the self? Journal of Homosexuality, 40(2), 31-48. https://doi.org/10.1300/J082v40n02_03

Wakil, S. P., Siddique, C. M., \& Wakil, F. A. (1981). Between two cultures: A study in socialization of children of immigrants. Journal of Marriage and Family, 43(4), 929-940. https://doi.org/10. 2307/351349

Wright, J. L. W., Adams, H. E., \& Bernat, J. (1999). Development and validation of the Homophobia Scale. Journal of Psychopathology and Behavioral Assessment, 21(4), 337-347. https://doi.org/10. 1023/a:1022172816258

Publisher's note Springer Nature remains neutral with regard to jurisdictional claims in published maps and institutional affiliations. 